the leading cause followed by motor vehicle-related injury. If the focus of the analysis is on injuries that result in hospitalisation, then adverse events and fall-related injury are the most frequent cause.

The analyses in the NSW Injury Profile also provide different pictures of the at-risk age and gender groups. The at-risk age and gender groups are similar for injury deaths and hospitalisations when looking at all injury mechanisms combined, but change when an injury mechanism is analysed separately, as illustrated by Table 3 .

The NSW Injury Profile depicts only the 'tip of the iceberg' regarding the effects that injury has on the people of NSW. Beyond the data presented in this article are the scores of injuries seen and treated every day by general practitioners, which are not recorded by existing systems of data collection. In the case of injuries that are captured in current systems of data collection (that is, deaths, hospitalisations, emergency department visits to some hospitals, and ambulance service calls), information describing the events that lead to the injury is often unavailable. This has resulted in large gaps in the data that are required to inform steps to prevent certain types of injury. Substantial gains in the field of injury prevention rest with rectifying these gaps in the available data.

\section{CONCLUSION}

This profile of injury in NSW has provided insight into the patterns of injury. The information will be used by the NSW Injury Risk Management Research Centre to further examine different mechanisms of injury, to develop targeted research plans to address areas of concern, and to identify approaches to injury prevention activities.

A copy of the NSW Injury Profile is available at www.irmrc.unsw.edu.au.

\title{
NSW INJURY RISK MANAGEMENT RESEARCH CENTRE
}

\section{Ann Williamson}

NSW Injury Risk Management Research Centre

University of New South Wales

The Injury Prevention and Policy Unit of the NSW Department of Health has developed a partnership with the NSW Roads and Traffic Authority, the Motor Accidents Authority, and the University of New South Wales, to establish the NSW Injury Risk Management Research Centre (IRMRC) at the University of New South Wales. This article briefly describes the aim, initiatives, activities, and research projects of the IRMRC.

\section{AIM OF THE IRMRC}

The aim of the IRMRC is to undertake and facilitate research on the prevention of all types of injury, and to develop effective solutions for problems associated with injury. The Centre provides industry and other agencies with a single point of access to expertise in a wide range of disciplines in the field of injury risk management.

\section{INITIATIVES OF THE IRMRC}

The IRMRC has three major initiatives:

- to identify and negotiate access rights to all relevant injury databases in order to conduct data mining and data analysis activities that will assist in the identification and subsequent monitoring of injury issues;

- to develop a database on injury in NSW. This is fundamental to determining the direction of the IRMRC's research activities and would also provide valuable advice to stakeholders and the public;

- to conduct research and to work in partnership with other researchers to encourage investigation of areas most likely to reduce the frequency and/or severity of injury.

\section{ACTIVITIES OF THE IRMRC}

The core activities of the IRMRC are to:

- establish a comprehensive information system;

- collate injury data across agencies;

- analyse data and provide advice for prevention;

- provide a clearing house for expertise;

- review and analyse literature in the field;

- research causes and control of injury;

- assist organisations to improve injury data collections.

\section{RESEARCH AREAS OFTHE IRMRC}

Some examples of the research areas associated with the identification, analysis and management of injury risks being coordinated by the IRMRC are: 
- establishing the context of the risk of injury, which includes the particular objectives of the partner organisations that sponsor the Centre and of other potential funding bodies;

- identifying and analysing sources of the risk of injury;

- analysing the causes of injury, the outcomes of injury, and the incidence of injury;

- developing risk assessment methodologies in the area of injury prevention;

- researching the risk of injury, which support recommendations for intervention strategies;

- evaluating levels of risk and appropriate intervention strategies, taking into account levels of risk, costs and benefits, and political constraints;

- evaluating injury control programs;

- monitoring risks and risk control strategies.

The IRMRC undertakes:

- long-term research relating to the management of the risks of injury, whether it is applied (with practical application) or fundamental (original research that investigates hypotheses);

- the acquisition of high quality information relating to the management of the risk of injury, from existing data sources, using the expertise of epidemiologists, statisticians, computer scientists, behavioural scientists, and engineers;

- the trial and evaluation of strategies to prevent injuries;

- short-term research for industry or government leading to policy and program advice;

- short-term research including data searches.

Population-based databases that the IRMRC currently has access to include:

- NSW Department of Health Inpatient Statistical Collection;
- Australian Bureau of Statistics Mortality data;

- NSW Roads and Traffic Authority Traffic Accident Database system;

- Motor Accidents Authority compensation database;

- NSW Health Survey;

- WorkCover NSW workers' compensation data.

Current projects include:

- NSW Injury Profile, with an analysis of patterns of injury for the most recent five year period (19951999) by age, gender, and injury mechanism;

- projects using the NSW Department of Health's Inpatient Statistical Collection, such as an analysis of work-related injury; further analysis of poisoningrelated injury, and an estimate of the cost of injury to government.

- an analysis of road crash data, 1992-2000, and a community attitude survey regarding road safety in NSW, for the NSW Road Safety Taskforce.

Other projects include: studies of coronial reports of fatalities of childen aged under six years in motor vehicle accidents and by drowning; an analysis of road crashes involving heavy motor vehicles; a trial of a minimum dataset on water safety; and a study of the effects of night and day driving on fatigue and performance.

Future projects planned by the IRMRC include: the development of an on-line injury data warehouse; the linkage of population datasets (for example, hospital and road crash, hospital and workers' compensation); and an in-depth analysis of injury causation and injury interventions.

Further information about the IRMRC and its current projects is available at www.irmrc.unsw.edu.au. 